\title{
Periosteal Sleeve Avulsion Fracture of the Quadriceps Tendon
}

\author{
Jordy van Sambeeck, BS ${ }^{1} \quad$ Elizabeth Arendt, MD $^{1} \quad$ Christopher Rud, BA ${ }^{1} \quad$ Jeffrey A. Macalena, MD ${ }^{1}$ \\ ${ }^{1}$ Department of Orthopaedic Surgery, University of Minnesota, \\ Minneapolis, Minnesota \\ J Knee Surg Rep 2016;2:e8-e10.

\begin{abstract}
Address for correspondence Jeffrey A. Macalena, MD, Department of Orthopaedic Surgery, University of Minnesota, 2450 Riverside Avenue, Suite R200, Minneapolis, MN 55454 (e-mail:
\end{abstract} \\ maca0049@umn.edu).
}

\author{
Abstract \\ Keywords \\ - sleeve avulsion \\ - sleeve \\ - quadriceps \\ - rupture
}

Sleeve fractures at the superior pole of the patella have been previously described in the literature. We present the case of a 15-year-old girl who sustained a patellar sleeve avulsion of the quadriceps tendon 1 month following patellar dislocation without surgical treatment. Open repair of quadriceps tendon was performed with subsequent union. Arthrofibrosis of the knee was noted; two subsequent manipulations and adhesiolysis were performed. At 18-month postinjury, normal motion and strength were present. Special attention to postoperative recovery of range of motion is imperative for good clinical outcome after periosteal sleeve avulsion fractures of the patella.
We present a 15-year-old girl with a periosteal sleeve avulsion fracture following a recurrent patellar dislocation. Sleeve fracture of the superior patellar pole has been described in patients with a history of patella dislocation and as a complication of surgery for patella instability. ${ }^{1}$

The patient's guardian provided consent regarding publication of this case report.

\section{Case Description}

A 15-year-old girl presented with a primary lateral patella dislocation of her left knee after she slipped off a starting block and struck the medial aspect of her knee. No reduction was required, the patellar dislocation spontaneously reduced with gentle knee extension prior to evaluation. Patellofemoral anatomy measurements demonstrated an Insall-Salvati ratio of 1.5, a Caton-Deschamps index of 1.6, and a tibial tubercletrochlear groove distance of $16 \mathrm{~mm}$. Initial radiographs were negative for fracture or loose bodies. A magnetic resonance imaging (MRI) demonstrated medial patellofemoral ligament rupture from its patellar origin, but neither chondral injuries nor damage to the superior patellar pole or quadriceps tendon. Conservative management consisted of weight bearing as tolerated, utilization of a patellar stabilization brace, and a graduated physical therapy (PT) program.

Patient progressed satisfactorily with conservative management. The patient's range of motion (ROM) profile had normalized, gait was nonantalgic, strengthening had returned, and a return to sports-specific activities was initiated. Twenty-seven days after index injury, she landed awkwardly on a trampoline with her left knee in flexion and presented with severe pain of the knee. The patient returned to jumping on a trampoline without clearance, no bracing was utilized for this activity. Examination revealed effusion, tenderness with palpation along the lateral and medial border and profound apprehension with lateral translation of the patella, passive ROM from 0 to 45 to 100 degrees of flexion, and inability to perform a straight leg raise. Standard radiographs (- Figs. 1a, b, and $\mathbf{2}$ ) demonstrated an osteochondral fragment along the medial patellar border on sunrise view, and superior to the patella on lateral view without anterior patellar tilt. This was interpreted as an osteochondral fragment from a second patellar dislocation. Repeat MRI demonstrated complete disruption of the quadriceps tendon insertion at the superior patellar pole with an associated avulsion of the periosteal sleeve, without evidence of fracture or other intra-articular pathology. Surgical repair was offered. received

October 8, 2015

accepted after revision

October 17, 2016

published online

January 6, 2017
Dol http://dx.doi.org/

$10.1055 / \mathrm{s}-0036-1597586$. ISSN 2326-2729.
Copyright (c) 2016 by Thieme Medical

Publishers, Inc., 333 Seventh Avenue, New York, NY 10001, USA.

Tel: +1(212) 584-4662.
License terms

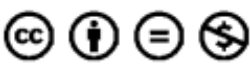




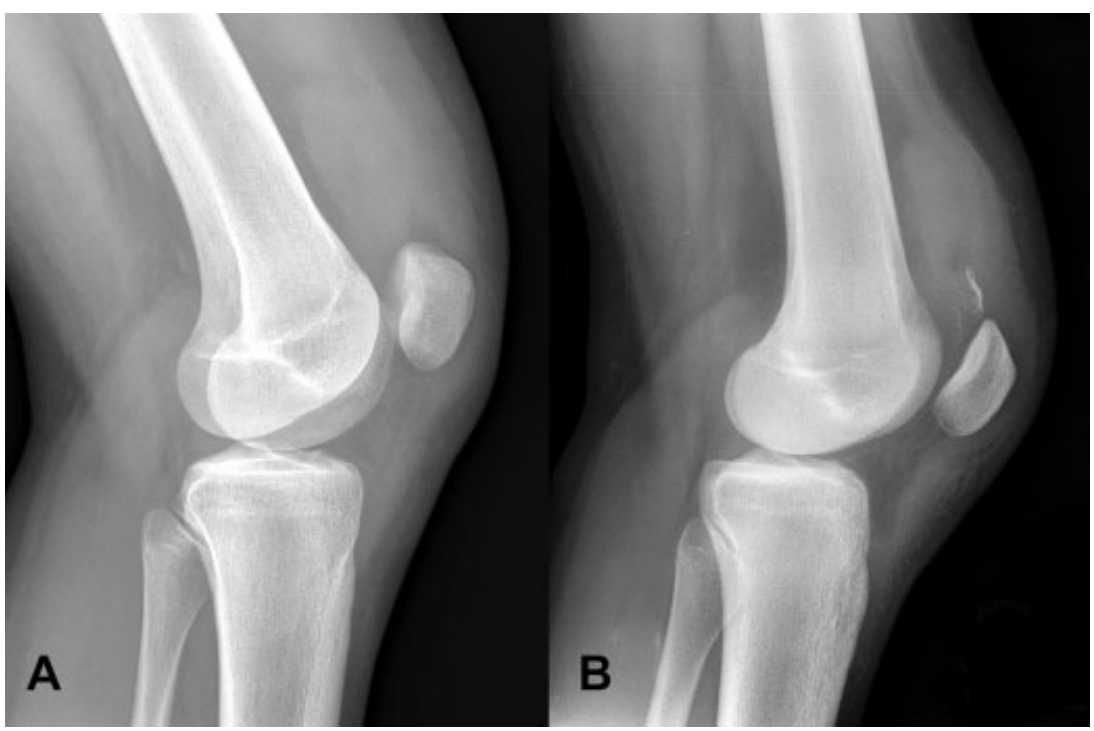

Fig.1 Lateral radiograph demonstrating (A) initial lateral radiograph after primary patella dislocation and (B) radiograph following the second trampoline injury with calcified fragment proximal to the patella.

Transosseous technique was utilized using four No. 2 Fiberwire locking Krackow sutures (Arthrex, Naples, FL) placed in the quadriceps tendon, two on each side of a vertical split in the tendon. These nonresorbable sutures were placed longitudinally through two medial and two lateral patellar tunnels (-Fig. 3). Avulsed periosteum was anatomically repaired after tensioning the sutures. Medial and lateral retinaculum repair was completed with No. 2 Fiberwire suture. A 2-mm drill bit was used for the longitudinal patellar drill holes. Fibrotic scar tissue was noted in the anterior chamber along the fat pad, anterior to the intracondylar notch. The quadriceps was mobilized and subcutaneous releases were performed. Postrepair ROM was 0 to 90 degrees. The knee was placed in a hinged brace in full extension and patient was admitted for pain control.

\section{Outcomes}

At 1 week, supervised PT started. A patient-controlled flexion machine (Flexionator, ERMI, Inc., Atlanta, GA) was started as she noted onset of stiffness and loss of a few degrees of

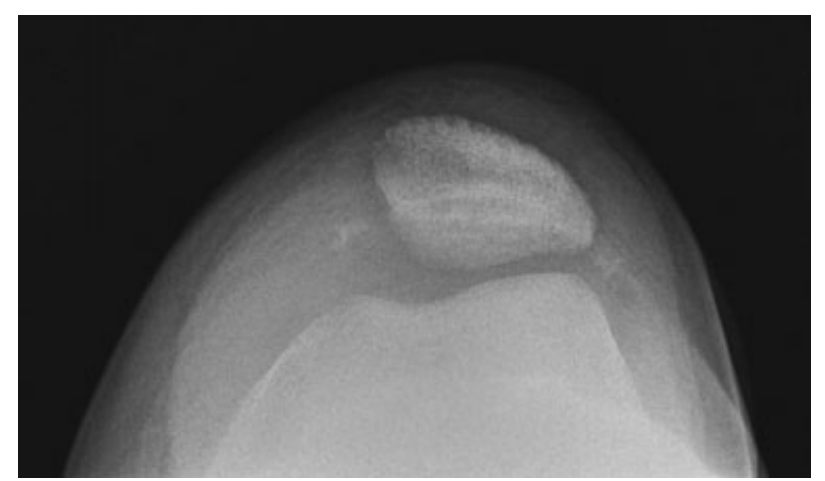

Fig. 2 Sunrise view following trampoline injury demonstrating an osteochondral fragment along the medial patellar border. flexion (ROM 0-0-40). At 5 weeks, multimodal therapy for pain control was given. Follow-up radiographs show maintenance of reduction.

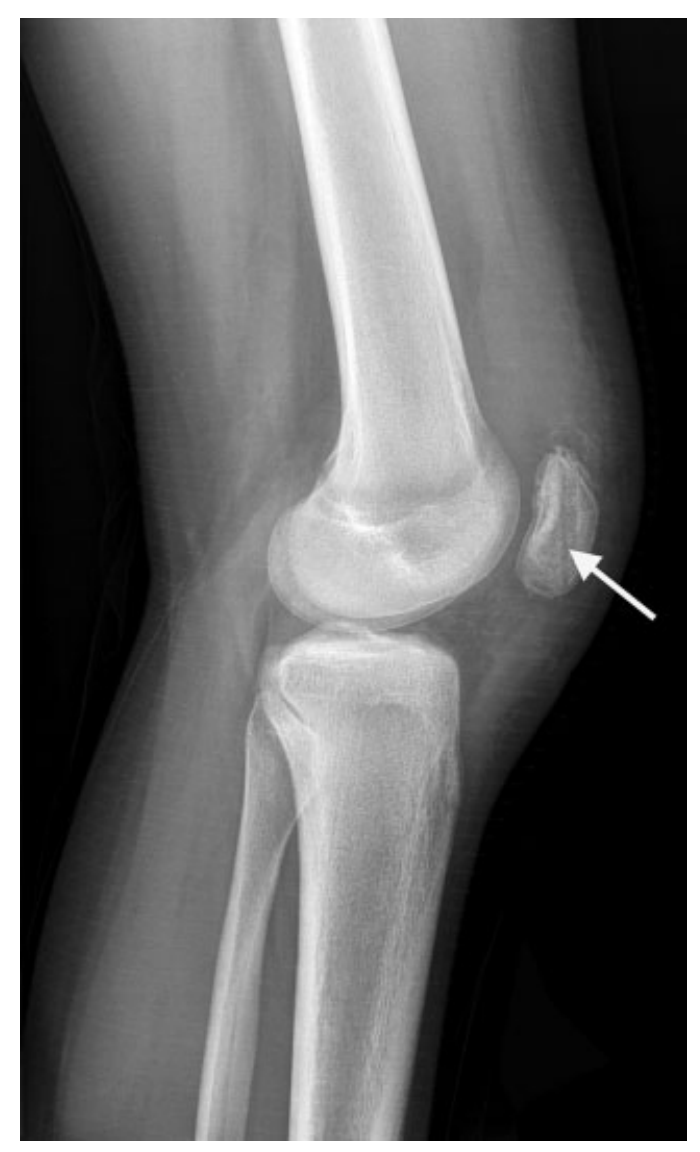

Fig. 3 Lateral radiograph 2 months postoperatively demonstrates repair of the periosteal sleeve avulsion of the quadriceps tendon. The arrow denotes the longitudinal tunnels through the patella. The reduced periosteum is seen well opposed to the anterior patella. 
At 8 weeks postoperative, flexion was limited to 45 degrees. Arthroscopic lysis of adhesions and manipulation under anesthesia was performed which demonstrated extensive arthrofibrosis in the medial and lateral gutters and complete loss of the suprapatellar pouch. Postoperatively, a continuous passive motion (CPM) machine was utilized to maintain ROM after release and PT continued.

At 8 weeks following the arthroscopic lysis of adhesions (16 weeks following repair), ROM had increased to 80 degrees but had again plateaued. Arthroscopic lysis of adhesions was again performed. An epidural was left in place and she was placed into a CPM machine at 0 to 70 to 120 degrees. Six weeks following the second lysis of adhesions (22 weeks following repair), excellent progress to 110 degrees of flexion was achieved. The patient was able to resume marching band and was cleared for all activities. At 12 months, ROM was 0 to 0 to 134 degrees on the left versus 3 to 0 to 147 degrees on the right knee. At 18 months postoperatively, she had returned to all desired activities without limitations.

\section{Discussion}

The differential diagnosis includes patellar dislocation with osteochondral fracture, sleeve avulsion fracture, and displaced osteochondritis dissecans. An MRI is useful to confirm the diagnosis. The presence of a new loose body on plain radiography, evidence of recurrent patellar instability, or an inability to perform a straight leg raise is indications to pursue an MRI.

Sleeve fracture of the patella is a traumatic avulsion of the lower or upper pole. The avulsed fragment contains articular cartilage on the deep surface and cartilage and periosteum on the subcutaneous surface. ${ }^{2}$ Sleeve fractures are attributed to a forceful contraction of the quadriceps against a flexed knee. $^{2,3}$ In this case, the mechanism of injury was an eccentric load on a flexed knee.

The superior pole sleeve avulsion fracture can easily be missed radiographically. A small fragment of bone adjacent to the proximal patellar pole might be the only radiographic finding; there might even be no bony damage visible. ${ }^{3-5}$ Anterior tilt of the patella has been suggested as a characteristic feature of superior pole sleeve fractures. ${ }^{1}$ MRI can be helpful in diagnosing this injury. In our case, the only radiographic finding was the osteochondral fragment. MRI demonstrated avulsion of the periosteal sleeve and a complete disruption of the extensor mechanism, and explained the origin of the fragment. This emphasizes the value of MRI in challenging cases for diagnosing this injury.

Nonoperative management is usually recommended for displacement less than $2 \mathrm{~mm}^{3}$ With more than $2 \mathrm{~mm}$ displacement, surgery with anatomical repair and fixation is recommended. In previous published cases, the period of immobilization after surgery is between 4 and 6 weeks, followed by ROM exercises in all except one report. Usually, full knee ROM with adequate strength was achieved in 8 to 16 weeks. ${ }^{3}$ It can be expected to obtain full motion and good strength with full return to activities. ${ }^{3,5}$ Since superior pole sleeve avulsion is a rare injury, there are no large studies evaluating different rehabilitation protocols and thus there is no evidence-based protocol available. Therefore, recommendations about such a protocol can only be based on results of published case reports or series.

Surgical complications of open repair have not been reported in the literature. Gettys et al mentioned possible complications of surgical repair of patella fractures in general including loss of fixation, stiffness, malunion, hardware irritation, and pain. ${ }^{3}$ Our case demonstrates that arthrofibrosis can be a significant complication of open repair of sleeve fracture and emphasizes the need for close patient monitoring and supervision, especially early in rehabilitation.

Patellar sleeve avulsion fractures require a high degree of clinical suspicion for correct diagnosis. After surgical repair of her injury, our patient struggled with limited flexion postoperatively, adequate pain management proved difficult. After two manipulations with adhesiolysis, ultimately the patient returned to all activities 18 months after her injury.

Key learning points of this case are the importance of close supervision to prevent complications after surgical treatment of sleeve fracture, especially arthrofibrosis, and the importance of sufficient pain control after surgical procedures of the knee for maximum rehabilitative effort.

\section{Note}

Institutional review board approval was obtained for this case report and from the subject.

\section{Funding}

No funding was received for this study.

\section{References}

1 Kumar K, Knight DJ. Sleeve fracture of the superior pole of patella: a case report. Knee Surg Sports Traumatol Arthrosc 2005;13(4): 299-301

2 Van Isacker T, De Boeck H. Sleeve fracture of the upper pole of the patella: a case report. Acta Orthop Belg 2007;73(1):114-117

3 Gettys FK, Morgan RJ, Fleischli JE. Superior pole sleeve fracture of the patella: a case report and review of the literature. Am J Sports Med 2010;38(11):2331-2336

4 Bates DG, Hresko MT, Jaramillo D. Patellar sleeve fracture: demonstration with MR imaging. Radiology 1994;193(3):825-827

5 Hunt DM, Somashekar N. A review of sleeve fractures of the patella in children. Knee 2005;12(1):3-7 